Accepted to ApJ

\title{
THE SPATIAL DISTRIBUTIONS OF RED AND BLUE GLOBULAR CLUSTERS IN MAJOR DRY MERGER REMNANTS
}

\author{
Min-Su Shin \\ Princeton University Observatory, Peyton Hall, Princeton, NJ 08544-1001 \\ msshin@astro.princeton. edu \\ and \\ Daisuke Kawata \\ The Observatories of the Carnegie Institution of Washington, 813 Santa Barbara Street, \\ Pasadena, CA 91101 \\ Swinburne University of Technology, Hawthorn VIC 3122, Australia \\ dkawata@ociw.edu
}

\begin{abstract}
Using high-resolution N-body simulations, we examine whether a major dry merger mitigates the difference in the radial density distributions between red and blue globular clusters (GCs). To this end, we study the relation between the density slope of the GCs in merger progenitors and that in a merger remnant, when the density distribution is described by $n_{\mathrm{GC}} \propto r^{-\alpha}$. We also study how our results depend on the merger orbit and the size of the core radius of the initial GC density distribution. We find that a major dry merger makes the GC profile flatter, and the steeper initial GC profile leads to more significant flattening, especially if the initial slope is steeper than $\alpha \sim 3.5$. Our result suggests that if there is a major dry merger of elliptical galaxies whose red GCs have a steeper radial profile than the blue GCs, as currently observed, and their slopes are steeper than $\alpha \sim 3.5$, the difference in the slopes between two populations becomes smaller after dry mergers. Therefore, the observed slopes of red and blue GCs can be a diagnostic of the importance of dry merger. The current observational data show that the red and blue GCs have more comparable and shallower slopes in some luminous galaxies, which may indicate that they have experienced dry mergers.
\end{abstract}


Subject headings: galaxies: elliptical - galaxies: evolution - galaxies: star clusters - globular clusters: general

\section{Introduction}

Globular clusters (GCs) in elliptical galaxies have been intensively studied in consideration of explaining the formation of both their host elliptical galaxies and GCs themselves (see Brodie \& Strader 2006, for a review). GCs are attractive as tracers of the star formation history of their host galaxies (e.g. Yi et al. 2004; Strader et al. 2006), because some properties of GC systems are correlated with the properties of their host galaxies (e.g., Brodie \& Huchra 1991; Djorgovski \& Santiago 1992). It is thought that the formation of GCs is triggered by starburst accompanying gas-rich galaxy merging (e.g. Schweizer 1987; Ashman \& Zepf 1992) or starburst that might happen with multiple dissipational collapses (Forbes et al. 1997). Forming young star clusters are found and they are expected to become star clusters like current old GCs in local galaxy mergers (Schweizer 2006).

An important aspect of GC systems in elliptical galaxies is a color bimodality (e.g., Zepf \& Ashman 1993; Geisler et al. 1996; Gebhardt \& Kissler-Patig 1999; Larsen et al. 2001; Peng et al. 2006). It is also found that red GCs are more centrally concentrated than blue GCs (e.g. Forte et al. 2005; Bassino et al. 2006a; Tamura et al. 2006), which could put additional constraints on their formation scenario (Bekki et al. 2002). The radial profile of each GC subpopulation is well described by a power-law distribution, especially at the outer radii, and the red GCs have a steeper slope than the blue GCs. Although the origin of this color bimodality is still uncertain, it is probably closely related to the formation history of their host elliptical galaxies (e.g., Yoon et al. 2006; Strader et al. 2007; Kundu \& Zepf 2007). Classically, three scenarios have been proposed to explain the color bimodality; major gas-rich mergers, in situ formation of multiple dissipational collapses, and dissipationless accretion. An explanation suggested by Ashman \& Zepf (1992) is that red GCs are metalrich clusters which might be formed by gas-rich disk-disk mergers. Therefore, the red GCs might be younger than blue GCs. Meanwhile, Forbes et al. (1997) explain that blue GCs might be formed in the first stage of dissipational collapse and red GCs might be formed after the truncation of the blue GC formation. Another explanation given by Coté et al. (1998) includes accretion of blue GCs from small galaxies into the already formed red GCs. More recently, Beasley et al. (2002) demonstrate that the bimodality can be explained in elliptical galaxy formation based on a hierarchical clustering scenario.

On the other hand, recent research suggests that in the late stage of evolution, early-type galaxies might have experienced dry merging where merger progenitors do not have much 
gas, nor accompany star formation. The number density evolution of red galaxies has been discussed in observations of COMBO-17 (Bell et al. 2004) and DEEP2 surveys (Faber et al. 2005), and such studies suggest that the density change can be understood by the dominance of dry merging after $z \lesssim 1$ (but see also Yamada et al. 2005; Cimatti et al. 2006; Bundy et al. 2007; Scarlata et al. 2007). The evolution of galaxy clustering also implies late effects on the evolution of massive red galaxies from dry merging (White et al.|2007). Moreover, the observations show that dry merging does occur (van Dokkum 2005; Tran et al. 2005; Rines et al. 2007), while the observed features of galaxies are well explained in cosmological simulations (e.g. Kawata et al. 2006). Recent theoretical studies of dry merger simulations of ellipticals show that merger remnants maintain their properties on the fundamental plane and other scaling relations (e.g. Nipoti et al. 2003; Boylan-Kolchin et al. 2005; Robertson et al. 2006; Ciotti et al. 2007). The dry merging of binary ellipticals also can explain the formation of boxy-type ellipticals (Naab et al. 2006).

Bekki \& Forbes (2006) demonstrate that the observed correlation between a spatial distribution of GCs and the total luminosity of ellipticals can be explained by sequential dissipationless major mergers, because the radial density profile of GCs progressively flatten after each major dry merger. This study raises the important question of whether or not the slopes of the density profiles of red and blue GCs persist after major dry merging. For example, we now consider that the density profiles of red and blue GCs in progenitor elliptical galaxies are described by $n_{\mathrm{GC}, \text { red }} \propto r^{-\alpha_{\mathrm{p} \text {,red }}}$ and $n_{\mathrm{GC} \text {,blue }} \propto r^{-\alpha_{\mathrm{p}, \text { blue }}}$, and these profiles in a major dry merger remnant become $n_{\mathrm{GC}, \text { red }} \propto r^{-\alpha_{\mathrm{r}, \text { red }}}$ and $n_{\mathrm{GC} \text {,blue }} \propto r^{-\alpha_{\mathrm{r}, \mathrm{blue}}}$. The current observations suggest that $\alpha_{\mathrm{r}, \text { red }}>\alpha_{\mathrm{r} \text {,blue }}$. However, if a dry merger flattens the red GC density profile more than the blue GC density profile, $\alpha_{\mathrm{p}, \text { red }}-\alpha_{\mathrm{r}, \text { red }}>\alpha_{\mathrm{p} \text {,blue }}-\alpha_{\mathrm{r} \text {,blue }}$, in the remnant galaxy the difference between $\alpha_{\mathrm{r}, \text { red }}$ and $\alpha_{\mathrm{r}, \mathrm{blue}}$ becomes smaller. In this case, the observed difference in the slopes of the density profiles of red and blue GCs in nearby ellipticals can be a valuable diagnostic for the importance of the dry merger in the evolution of ellipticals. To clarify this issue, the question becomes how the flattening of GC profiles during dry merging depends on the initial distributions of the GCs.

We use numerical simulations of major dry mergers to study the dependence of GC distributions in merger remnants on the initial distributions in merger progenitors. Then, we can compare $\alpha_{\mathrm{p} \text {,red }}-\alpha_{\mathrm{r} \text {,red }}$ and $\alpha_{\mathrm{p} \text {,blue }}-\alpha_{\mathrm{r} \text {,blue }}$, for different sets of $\alpha_{\mathrm{p} \text {,red }}$ and $\alpha_{\mathrm{p} \text {,blue }}$. Since major dry mergers between two equal-mass merger progenitors must leave the most significant effects on merger remnants, compared with minor mergers, we study only equalmass mergers in this paper. In $\S 2$, we explain details of our dry merger simulations and initial GC distributions. The changes in GC spatial distributions due to major mergers are shown in $\S 3$. We discuss the implication of our results in $\S 4$ that is followed by conclusion. 


\section{Simulation and parameters}

Our galaxy merger simulations are carried out with a Tree N-body code, GCD+ Kawata \& Gibson 2003). We model a merger progenitor elliptical galaxy which consists of a stellar bulge and a dark matter (DM) halo. The stellar component is assumed to follow the Hernquist (1990) density profile,

$$
\rho_{*}(r)=\frac{M_{*}}{2 \pi} \frac{a}{r} \frac{1}{(r+a)^{3}} .
$$

We set the total stellar mass $\mathrm{M}_{*}=10^{11} \mathrm{M}_{\odot}$. Following Boylan-Kolchin et al. (2006), we use an empirical relation from Shen et al. (2003),

$$
R_{\mathrm{e}}=4.16\left(\frac{M_{*}}{10^{11} M_{\odot}}\right)^{0.56} \mathrm{kpc} .
$$

For the Hernquist profile, $R_{\mathrm{e}}$ can be described with $R_{\mathrm{e}}=1.8153 a$.

The initial DM halo is assumed to follow the NFW density profile (Navarro et al. 1997);

$$
\rho=\frac{3 H_{0}^{2}}{8 \pi G}\left(1+z_{0}\right)^{3} \frac{\Omega_{0}}{\Omega(z)} \frac{\delta_{c}}{c x(1+c x)^{2}},
$$

where $c$ is a concentration parameter, and $x=r / r_{200}$ is a radius normalized by $r_{200}$ which is the radius of a sphere whose mean interior density is $200 \rho_{\text {crit }}$. The characteristic density $\delta_{c}$ is described as

$$
\delta_{c}=\frac{200}{3} \frac{c^{3}}{\ln (1+c)-c /(1+c)} .
$$

The radius of $r_{200}$ is linked with $\mathrm{M}_{200}$, the mass within the radius, with

$$
r_{200}=1.63 \times 10^{-2}\left(\frac{M_{200}}{h^{-1} M_{\odot}}\right)^{1 / 3}\left[\frac{\Omega_{0}}{\Omega\left(z_{0}\right)}\right]^{-1 / 3}\left(1+z_{0}\right)^{-1} h^{-1} \mathrm{kpc} .
$$

We assume $c=10, z_{0}=0, H_{0}=70 \mathrm{~km} \mathrm{~s}^{-1} \mathrm{Mpc}^{-1}$. The final DM density profile takes into account adiabatic contraction, following the formula suggested by Blumenthal et al. (1986). Following Springel \& White (1999), we apply an exponential cut-off for the dark matter density profile at $r>r_{200}$. We define the total mass of the DM component as the DM mass within $2 r_{200}$, where the density is low enough. The total mass of the DM component is set to be $\mathrm{M}_{\mathrm{DM} \text {,tot }}=\mathrm{M}_{\mathrm{NFW}}\left(2 r_{200}\right)-\mathrm{M}_{*}$, where $\mathrm{M}_{\mathrm{NFW}}\left(2 r_{200}\right)$ is calculated from the exponentially truncated NFW profile with $\mathrm{M}_{200}=10^{12} \mathrm{M}_{\odot}$. The DM and stellar components are composed of $10^{5}$ and $10^{4}$ particles, respectively, so that the particles for the different components have roughly same mass. As a result, the particle mass and softening length are $\sim 10^{7} \mathrm{M}_{\odot}$ and $0.58 \mathrm{kpc}$, respectively. We also run one simulation with half number of particles in order to verify convergence of our results. 
The velocity dispersions for both DM and stellar components are assumed to be isotropic, $\sigma_{r}=\sigma_{\theta}=\sigma_{\phi}$, and follow the Jeans equation. Then, the velocity dispersion can be written as

$$
\rho(r) \sigma_{r}^{2}(r)=G \int_{r}^{\infty} \rho(s) M(<s) \frac{\mathrm{d} s}{s^{2}},
$$

as shown in Mamon \& Eokas (2005). In our simulations, the two merger progenitors have exactly the same distributions of DM and star particles for simplicity.

Three different merger orbits are studied in our simulations as given in Table 1. From a fixed initial separation of $400 \mathrm{kpc}$, we have chosen three parabolic orbits (Barnes 1992); (A) head-on collision; (B) a close encounter with pericentric distance of the orbit, $r_{\text {peri }}=5$ kpc; and (C) a wider encounter with $r_{\text {peri }}=50 \mathrm{kpc}$. All simulations are stopped after 8 Gyrs even though the merger remnants are fully relaxed within $300 \mathrm{kpc}$ within 6 Gyrs.

For simplicity, we assume that the GCs are spherically distributed and that the number density follows the following radial profile:

$$
n_{\mathrm{GC}}(r) \propto \frac{1}{\left(r^{2}+r_{c}^{2}\right)^{\alpha_{\mathrm{p}} / 2}},
$$

where $r_{\mathrm{c}}$ is a core radius. We include the core, because the observed GC density profiles suggest the existence of the core (Forbes et al. 1996). We also examine how the initial core size affects the slope at the outer radii in a dry merger remnant. Here, instead of putting additional particles to trace the positions of GCs during the merger, we randomly select particles from DM particles, such that the selected GC particles follow the number density distribution of equation (77) as well as velocity dispersion distribution of equation (6). This sampling of GC particles has the advantage that we can simulate various different initial distributions without running a simulation several times, which enables us to study the detailed dependence of the flattening on the initial GC profiles.

After the merger, we analyze the number density profile of the selected GC particles. We are interested in how final slope of the GC density profile depends on the initial slope. We fit the profile of the number density at the radii between 10 and $100 \mathrm{kpc}$ by $n_{\mathrm{GC}} \propto r^{-\alpha_{\mathrm{r}}}$. This radial region is chosen arbitrarily. However, the important point is that we compare the slope at a fixed radial region throughout the paper. We consider initial density slopes between $\alpha_{\mathrm{p}}=2$ and 5 , and the two different core radii of $r_{\mathrm{c}}=2.5$ and $5 \mathrm{kpc}$ to see the effect of the initial core size on the final slope. For each initial GC profile, we sample 10,000 GC particles for each merger progenitor. Figure 1 demonstrates that our sampling well reproduces the input number density profile for GCs even at the outer radii. It also confirms that the distribution of velocity dispersions for the GCs is consistent with the equilibrium values from equation ( 6 ). 
We also test stability of the initial distribution of the selected GC particles by running a simulation of a single isolated galaxy with the sampled GC particles. After 8 Gyrs, the isolated single galaxy maintains the initial distribution of the GCs, as shown in Figure 2 .

\section{Results}

We present the projected density distribution of the stellar component of a merger remnant in Figure 3. We can see that there is a negligible effect from the difference in orbits on the distribution of stars in the merger remnant. We also fit the final stellar density profiles between $r=1$ and $40 \mathrm{kpc}$ with de Vaucouleurs profiles. In Figure 3, de Vaucouleurs profile describes well the projected distribution of the stellar component in all merger remnants from the three different orbits. The measured effective radius is close to $\sim 6.2 \mathrm{kpc}$ for all three simulations, which is similar to the results in Boylan-Kolchin et al. (2005).

In Figure 4 we show the final GC number density profile for the different models with $\alpha_{\mathrm{p}}=2,4$ and 5 for simulation B. We first focus on the results for simulation B, and discuss the orbital dependence later. For models of $\alpha_{\mathrm{p}}=4$ and 5 , the GC profile in the merger remnants is clearly flatter than the initial one. This result is consistent with what have been

found in similar dissipationless galaxy merger simulations (White 1978, 1980; Villumsen 1982, 1983; Duncan et al. 1983; White 1983; Bekki \& Forbes 2006). On the other hand, for the $\alpha_{\mathrm{p}}=2$ model there is not much change in the density slope of the GC distribution in a merger remnant. In addition, comparing models of $\alpha_{\mathrm{p}}=4$ and 5 , the difference between the initial and the final profile is more significant for the $\alpha_{\mathrm{p}}=5$ model. Hence, flattening of the $\mathrm{GC}$ radial profile due to a major dry merger clearly depends on the GC density profile in a progenitor galaxy.

Figure 5 summarizes our main results. We find that a higher $\alpha_{\mathrm{p}}$ leads to a larger $\alpha_{\mathrm{p}}-\alpha_{\mathrm{r}}$. In other words, the steeper initial GC profiles result in stronger flattening. The boundary between a significant and a weak flattening is around $\alpha_{\mathrm{p}} \sim 3.5$. In the next sections, we discuss the implication of our results for the evolution in the relative distributions of red and blue GCs during a major dry merger. First, we briefly examine the importance of the merger orbits and the initial core radii.

\section{1. $\quad$ Merger Orbits}

We find that the final GC density profile weakly depends on a merger orbit as shown in Figure 6. The found maximum difference of $\alpha_{\mathrm{r}}$ is $\sim 0.2$ for $\alpha_{p}=5$. As also highlighted in Fig- 
ure 7 of the three orbits, the simulation $\mathrm{C}$ causes the least flattening in the GC distribution. The highest angular momentum collision is not efficient in flattening GC distributions.

We also find that the density profile in the outer regions of merger remnants are more sensitive to the merger orbits than that in the inner region. In Figure 7, all three merger orbits do not produce any differences in $r<10 \mathrm{kpc}$ for an initial $\alpha_{\mathrm{p}}=5$ model. However, beyond $r \sim 10 \mathrm{kpc}$, the small difference of flattening begins to appear.

As a convergence test, we also run simulation $\mathrm{A}$ with a half number of particles, i.e, $5 \times 10^{4} \mathrm{DM}, 5 \times 10^{3}$ stellar, and $5 \times 10^{3} \mathrm{GC}$ particles. Figure 2 demonstrates that the low resolution simulation also provides a stable initial condition. As shown in Figure 8 , the low-resolution simulation leads to the consistent results to the high-resolution simulation. Thus, our results are not affected by the number of particles adopted.

\subsection{Dependence on the size of core radius}

In Figure 9, we compare the GC slopes after major dry mergers in the cases where the merger progenitors have the core radii of $r_{\mathrm{c}}=2.5$ and $5 \mathrm{kpc}$. Larger initial core radii result in stronger flattening. Therefore, it is clear that the flattening due to a dry merger depends on the initial core radius.

The size of the initial core radius is a more important factor for the final density slope than the merger orbit, as found in the comparison between Figure 6 and 10. In particular, the size of the initial core radius affects the steepening of the GC density profile differently, depending on $\alpha_{\mathrm{p}}$. The difference in $\alpha_{\mathrm{r}}$ between the different initial core radii is more significant for a larger $\alpha_{\mathrm{p}}$ model than for a smaller $\alpha_{\mathrm{p}}$. Hence it is another interesting issue to see in more detail how the initial core radius affects the flattening. However, in our method described in $\S 2$, it is difficult to make the initial distribution of GCs with a very small core. Moreover, simulations of a small $r_{\mathrm{c}}$ also need higher spatial resolution than we currently employ, and so we leave this question for future study.

\section{Discussion and Conclusion}

Our main result is that steeper initial GC profiles experience stronger flattening as shown in Figure 5. $\alpha_{\mathrm{p}} \approx 3.5$ is in boundary between strong and weak flattening. The results imply that if the initial slopes of both red and blue GCs are steeper than $\alpha_{\mathrm{p}} \approx 3.5$, the difference in the slopes between two populations of GCs will become much smaller, independent of merger orbits. In particular, even only one dry merger can make the slope 
flatter dramatically. Moreover, it also makes both slopes be around $\alpha_{\mathrm{r}} \approx 3.5-4.5$. For example, if $\alpha_{\mathrm{p} \text {,red }}=5$ and $\alpha_{\mathrm{p} \text {,blue }}=4$, Figure 5 suggests that $\alpha_{\mathrm{r}, \text { red }} \approx 4.3$ and $\alpha_{\mathrm{p}, \text { blue }} \approx 3.6$. The difference in the slope between red and blue GCs becomes significantly small. Therefore, the difference in the slopes of red and blue GCs can be a constraint of the number of major dry mergers.

On the other hand, if the initial GC distribution has a slope shallower than $\alpha_{\mathrm{p}} \approx 3.5$, the slope changes very little, and almost no change in the case of $\alpha_{\mathrm{p}} \approx 2$. Therefore, if the initial distributions of red and blue GCs in merger progenitors are steeper than $\alpha_{\mathrm{p}}=2$, and the galaxies experienced a number of major dry mergers, it leads the distributions of both red and blue GCs to become close to $\alpha_{\mathrm{r}} \sim 2$ for both red and blue GCs, and the difference in the slope of spatial distributions becomes difficult to be measured.

It is also worth noting that if the initial core size is larger for blue GCs than for red GCs, but their initial slopes are the same, a major dry merger can make the slope for the blue GCs shallower than for the red GCs, as shown in Figure 9, Therefore, the final slope is not a simple function of the initial slope.

Our results suggest that dry mergers make the slopes of the density profiles for red and blue GCs shallower and similar. Several studies (e.g. Kissler-Patig 1997; van den Bergh 1998; Forbes 2005; Lauer et al. 2007; Emsellem et al. 2007) claim that the various observed properties for ellipticals show a transition around $\mathrm{M}_{\mathrm{V}} \sim-21$, i.e. $\sim 10^{11} \mathrm{M}_{\odot}$. Some of these bimodalities have been interpreted in the picture of dry merger hypothesis for the growth of massive ellipticals (e.g. Capetti \& Balmaverde 2006). For example, Emsellem et al. (2007) show that slowly rotating ellipticals might be mainly affected by dry mergers. The slowly rotating ellipticals are more luminous than $\mathrm{M}_{\mathrm{B}} \approx-20.5$, while less luminous ellipticals are fast rotators. It may indicate that less luminous galaxies have not experienced any major dry mergers. Therefore, it is interesting to examine the observed distributions of red and blue GCs as a function of the stellar mass of ellipticals. If more luminous galaxies have experienced dry mergers, our results predict that the slopes for red and blue GCs in bright galaxies are shallower and similar. Unfortunately, the current observational samples are not enough to test it statistically. Below we provide some discussion based on the current limited measurements.

NGC 1399 is one of ellipticals whose spatial distributions of red and blue GCs are well-observed (Dirsch et al. 2003; Bassino et al. 2006a). When assuming $\mathrm{M} / \mathrm{L}_{\mathrm{V}}=5$ and $B-V=0.9$ for an elliptical galaxy, as used in Forbes (2005), the stellar mass of NGC 1399 is about $5 \times 10^{11} \mathrm{M}_{\odot}\left(\mathrm{M}_{\mathrm{B}}=-21.8\right)$ (Brodie \& Strader 2006). The derived power-law indices of projected radial density profiles are $1.9 \pm 0.06$ and $1.6 \pm 0.10$ for red and blue GCs, respectively (Bassino et al. 2006a). If the distributions are spherically symmetric, the 
three-dimensional radial distributions have power-law slopes of $\alpha \approx 2.9$ and $\approx 2.6$ for red and blue GCs, respectively. Note that the real slope might be slightly steeper, because the distribution of GCs is not infinite. The slope difference between red and blue GC distributions is $\Delta \alpha \approx 0.3$ while both GC populations show $\alpha \approx 3$. In addition to NGC 1399, NGC 1407 is a brightest group galaxy with $\mathrm{M}_{\mathrm{V}}=-21.86$, and has a bimodal color distribution of GCs. Forbes et al. (2006) report that the projected slopes of the red and blue GC density profiles are respectively $1.50 \pm 0.05$ and $1.65 \pm 0.29$, i.e., expected three dimensional slopes of $\alpha_{\text {red }}=2.50 \alpha_{\text {blue }}=2.65$, which are statistically the same as each other.

On the other hand, NGC 1374 and NGC 1379 are less luminous than NGC 1399, having $\mathrm{M}_{\mathrm{V}}=-20.4$ and -20.6 , respectively. The red and blue GCs of the two galaxies are studied in Bassino et al. (2006b). The derived projected density distributions of red and blue GCs in NGC 1374 have slopes of 3.2 and 2.3. Hence, the expected slopes in their three-dimensional density profiles are $\alpha_{\text {red }} \sim 4.2$ and $\alpha_{\text {blue }} \sim 3.3$. The $\Delta \alpha$ in NGC 1379 is $\sim 0.6$, and the red and blue GCs have projected power-law slopes of $\sim 2.9$ and $\sim 2.3$, respectively. The low-luminosity galaxies have a larger difference in the density slopes of the red and blue GCs, and their slopes are steeper than the more luminous galaxies discussed above. Combining with our results, these four sample suggests that luminous galaxies, like NGC 1399 and NGC 1407, may have experienced some dry mergers, while less luminous galaxies, such as NGC 1374 and NGC 1379, may have not been formed through dry mergers, which is consistent with a scenario that the significance of dry mergers depends on the stellar mass.

However, we also find some giant galaxy that does not follow the above trend. Recently, Tamura et al. (2006) measured the density profiles of red and blue GCs for giant ellipticals: M87 and NGC 4552. We have fitted the projected density profile in Figure 5 of their paper by a power-law profile, and the derived power-law index is 2.4 and 1.4 for red and blue GCs in M87 and 1.8 and 1.2 for red and blue GCs in NGC 4552, respectively. Although these giant ellipticals have relatively shallower slopes, the difference in slopes of red and blue GCs is significant. According to our result, these giant ellipticals are unlikely to have experienced significant dry mergers. Some giants might form without dry mergers, or some other factor, such as a number of minor mergers, might affect the slopes of GCs.

Again, so far there are not many studies which derive the density distributions of both red and blue GCs. This problem limits the application of our results to the current observational data. However, we expect that future observations will improve the statistics for nearby ellipticals, and our results will add an important framework to interpret the galaxies' evolutionary history. It will be also valuable to compare spatial distributions of blue and red GCs with other expected properties from dry mergers such as surface brightness profile. 
We thank Jeremiah P. Ostriker, James E. Gunn, and Patricia Sánchez-Blázquez for many fruitful discussions. We also thank Jenny E. Greene for careful reading of the manuscript, and Naoyuki Tamura for the data of M87 and NGC 4552. We thank the anonymous referee for helpful comments. M.-S.S. wishes to thank the Observatories of the Carnegie Institution of Washington for its hospitality. M.-S.S. was supported in part by the Korean Science and

Engineering Foundation Grant KOSEF-2005-215-C00056 funded by the Korean government (MOST). This research used computational facilities supported by NSF grant AST-0216105.

\section{REFERENCES}

Ashman, K. M., \& Zepf, S. E. 1992, ApJ, 384, 50

Barnes, J. E. 1992, ApJ, 393, 484

Bassino, L. P., Faifer, F. R., Forte, J. C., Dirsch, B., Richtler, T., Geisler, D., \& Schuberth, Y. 2006a, A\&A, 451, 789

Bassino, L. P., Richtler, T., \& Dirsch, B. 2006b, MNRAS, 367, 156

Beasley, M. A., Baugh, C. M., Forbes, D. A., Sharples, R. M., \& Frenk, C. S. 2002, MNRAS, 333, 383

Bekki, K., \& Forbes, D. A. 2006, A\&A, 445, 485

Bekki, K., Forbes, D. A., Beasley, M. A., \& Couch, W. J. 2002, MNRAS, 335, 1176

Bell, E. F., Wolf, C., Meisenheimer, K., Rix, H.-W., Borch, A., Dye, S., Kleinheinrich, M., Wisotzki, L., \& McIntosh, D. H. 2004, ApJ, 608, 752

Blumenthal, G. R., Faber, S. M., Flores, R., \& Primack, J. R. 1986, ApJ, 301, 27

Boylan-Kolchin, M., Ma, C.-P., \& Quataert, E. 2005, MNRAS, 362, 184

—. 2006, MNRAS, 369, 1081

Brodie, J. P., \& Huchra, J. P. 1991, ApJ, 379, 157

Brodie, J. P., \& Strader, J. 2006, ARA\&A, 44, 193

Bundy, K., Treu, T., \& Ellis, R. S. 2007, ApJ, 665, L5

Capetti, A., \& Balmaverde, B. 2006, A\&A, 453, 27 
Cimatti, A., Daddi, E., \& Renzini, A. 2006, A\&A, 453, L29

Ciotti, L., Lanzoni, B., \& Volonteri, M. 2007, ApJ, 658, 65

Coté, P., Marzke, R. O., \& West, M. J. 1998, ApJ, 501, 554

Dirsch, B., Richtler, T., Geisler, D., Forte, J. C., Bassino, L. P., \& Gieren, W. P. 2003, AJ, 125,1908

Djorgovski, S., \& Santiago, B. X. 1992, ApJ, 391, L85

Duncan, M. J., Farouki, R. T., \& Shapiro, S. L. 1983, ApJ, 271, 22

Emsellem, E., Cappellari, M., Krajnović, D., van de Ven, G., Bacon, R., Bureau, M., Davies, R. L., de Zeeuw, P. T., Falcón-Barroso, J., Kuntschner, H., McDermid, R., Peletier, R. F., \& Sarzi, M. 2007, MNRAS, 379, 401

Faber, S. M., Willmer, C. N. A., Wolf, C., Koo, D. C., Weiner, B. J., Newman, J. A., Im, M., Coil, A. L., Conroy, C., Cooper, M. C., Davis, M., Finkbeiner, D. P., Gerke, B. F., Gebhardt, K., Groth, E. J., Guhathakurta, P., Harker, J., Kaiser, N., Kassin, S., Kleinheinrich, M., Konidaris, N. P., Lin, L., Luppino, G., Madgwick, D. S., Noeske, K. M. K. G., Phillips, A. C., Sarajedini, V. L., Simard, L., Szalay, A. S., Vogt, N. P., \& Yan, R. 2005, ArXiv Astrophysics e-prints

Forbes, D. A. 2005, ApJ, 635, L137

Forbes, D. A., Brodie, J. P., \& Grillmair, C. J. 1997, AJ, 113, 1652

Forbes, D. A., Franx, M., Illingworth, G. D., \& Carollo, C. M. 1996, ApJ, 467, 126

Forbes, D. A., Sánchez-Blázquez, P., Phan, A. T. T., Brodie, J. P., Strader, J., \& Spitler, L. 2006, MNRAS, 366, 1230

Forte, J. C., Faifer, F., \& Geisler, D. 2005, MNRAS, 357, 56

Gebhardt, K., \& Kissler-Patig, M. 1999, AJ, 118, 1526

Geisler, D., Lee, M. G., \& Kim, E. 1996, AJ, 111, 1529

Hernquist, L. 1990, ApJ, 356, 359

Kawata, D., \& Gibson, B. K. 2003, MNRAS, 340, 908

Kawata, D., Mulchaey, J. S., Gibson, B. K., \& Sánchez-Blázquez, P. 2006, ApJ, 648, 969 
Khochfar, S., \& Burkert, A. 2006, A\&A, 445, 403

Kissler-Patig, M. 1997, A\&A, 319, 83

Kundu, A., \& Zepf, S. E. 2007, ApJ, 660, L109

Larsen, S. S., Brodie, J. P., Huchra, J. P., Forbes, D. A., \& Grillmair, C. J. 2001, AJ, 121, 2974

Lauer, T. R., Gebhardt, K., Faber, S. M., Richstone, D., Tremaine, S., Kormendy, J., Aller, M. C., Bender, R., Dressler, A., Filippenko, A. V., Green, R., \& Ho, L. C. 2007, ApJ, 664,226

Mamon, G. A., \& Łokas, E. L. 2005, MNRAS, 362, 95

Naab, T., Khochfar, S., \& Burkert, A. 2006, ApJ, 636, L81

Navarro, J. F., Frenk, C. S., \& White, S. D. M. 1997, ApJ, 490, 493

Nipoti, C., Londrillo, P., \& Ciotti, L. 2003, MNRAS, 342, 501

Peng, E. W., Jordán, A., Côté, P., Blakeslee, J. P., Ferrarese, L., Mei, S., West, M. J., Merritt, D., Milosavljević, M., \& Tonry, J. L. 2006, ApJ, 639, 95

Rines, K., Finn, R., \& Vikhlinin, A. 2007, ApJ, 665, L9

Robertson, B., Cox, T. J., Hernquist, L., Franx, M., Hopkins, P. F., Martini, P., \& Springel, V. 2006, ApJ, 641, 21

Scarlata, C., Carollo, C. M., Lilly, S. J., Feldmann, R., Kampczyk, P., Renzini, A., Cimatti, A., Halliday, C., Daddi, E., Sargent, M. T., Koekemoer, A., Scoville, N., Kneib, J.P., Leauthaud, A., Massey, R., Rhodes, J., Tasca, L., Capak, P., McCracken, H. J., Mobasher, B., Taniguchi, Y., Thompson, D., Ajiki, M., Aussel, H., Murayama, T., Sanders, D. B., Sasaki, S., Shioya, Y., \& Takahashi, M. 2007, ApJS, 172, 494

Schweizer, F. 1987, in Nearly Normal Galaxies. From the Planck Time to the Present, ed. S. M. Faber, 18-25

Schweizer, F. 2006, ArXiv Astrophysics e-prints

Shen, S., Mo, H. J., White, S. D. M., Blanton, M. R., Kauffmann, G., Voges, W., Brinkmann, J., \& Csabai, I. 2003, MNRAS, 343, 978

Springel, V., \& White, S. D. M. 1999, MNRAS, 307, 162 
Strader, J., Beasley, M. A., \& Brodie, J. P. 2007, AJ, 133, 2015

Strader, J., Brodie, J. P., Spitler, L., \& Beasley, M. A. 2006, AJ, 132, 2333

Tamura, N., Sharples, R. M., Arimoto, N., Onodera, M., Ohta, K., \& Yamada, Y. 2006, MNRAS, 373, 601

Tran, K.-V. H., van Dokkum, P., Franx, M., Illingworth, G. D., Kelson, D. D., \& Schreiber, N. M. F. 2005, ApJ, 627, L25

van den Bergh, S. 1998, ApJ, 492, 41

van Dokkum, P. G. 2005, AJ, 130, 2647

Villumsen, J. V. 1982, MNRAS, 199, 493

-. 1983, MNRAS, 204, 219

White, M., Zheng, Z., Brown, M. J. I., Dey, A., \& Jannuzi, B. T. 2007, ApJ, 655, L69

White, S. D. M. 1978, MNRAS, 184, 185

—. 1980, MNRAS, 191, 1P

White, S. D. M. 1983, in IAU Symposium, Vol. 100, Internal Kinematics and Dynamics of Galaxies, ed. E. Athanassoula, 337-344

Yamada, T., Kodama, T., Akiyama, M., Furusawa, H., Iwata, I., Kajisawa, M., Iye, M., Ouchi, M., Sekiguchi, K., Shimasaku, K., Simpson, C., Tanaka, I., \& Yoshida, M. 2005, ApJ, 634, 861

Yi, S. K., Peng, E., Ford, H., Kaviraj, S., \& Yoon, S.-J. 2004, MNRAS, 349, 1493

Yoon, S.-J., Yi, S. K., \& Lee, Y.-W. 2006, Science, 311, 1129

Zepf, S. E., \& Ashman, K. M. 1993, MNRAS, 264, 611 
Table 1. MERGER ORBITS

\begin{tabular}{cccc}
\hline \hline Simulation & $\Delta v(\mathrm{~km} / \mathrm{s})$ & $e$ & $r_{\text {peri }}(\mathrm{kpc})$ \\
\hline $\mathrm{A}$ & $(206,0.0,0.0)$ & 1.0 & 0.0 \\
$\mathrm{~B}$ & $(205,23,0.0)$ & 1.0 & 5.0 \\
$\mathrm{C}$ & $(193,73,0.0)$ & 1.0 & 50 \\
\hline
\end{tabular}

Note. - Eccentricity, $e$, and perigee distance, $r_{p e r i}$, are derived from the reduced twobody problem (e.g. Khochfar \& Burkert 2006) 

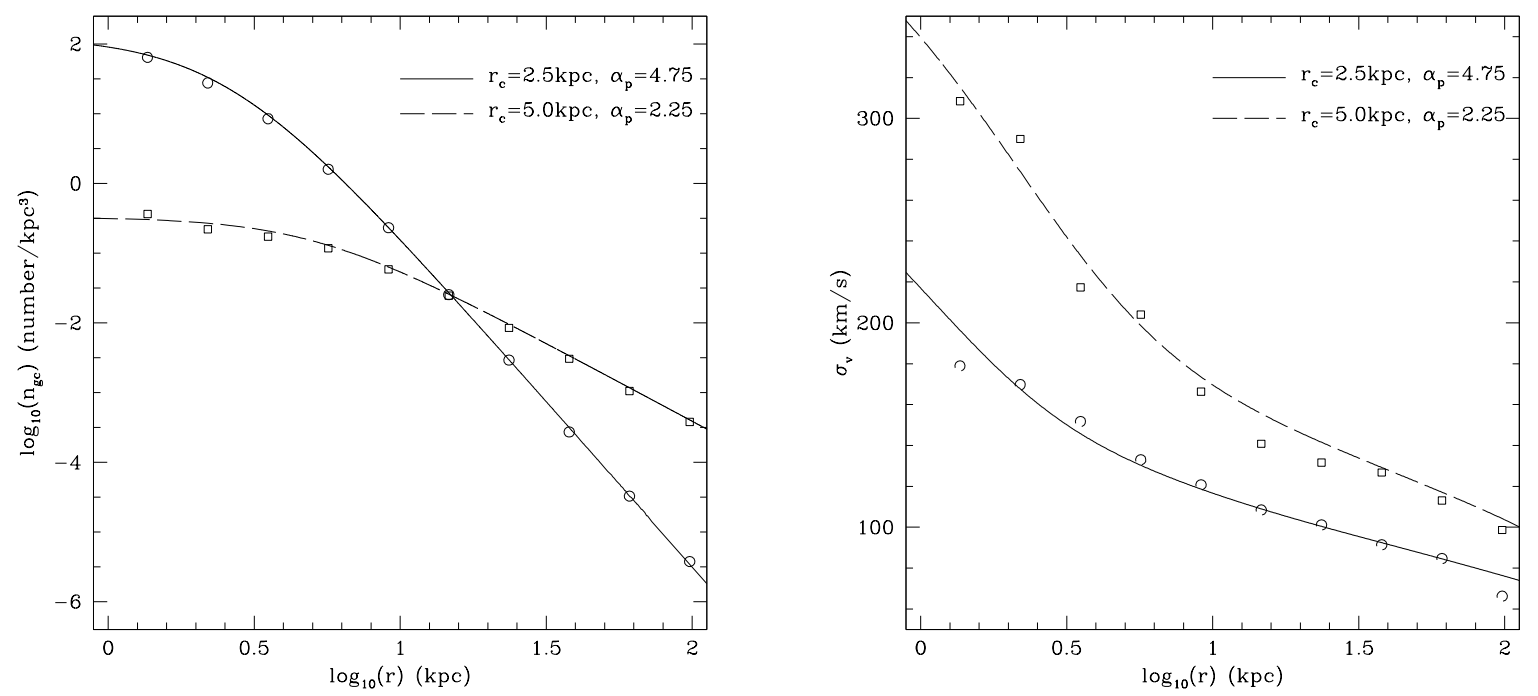

Fig. 1.- Initial number density (left) and velocity dispersion (right) profiles of GCs in merger progenitors. The input profiles of $r_{c}=2.5 \mathrm{kpc}$ and $\alpha_{p}=4.75$ are shown as solid lines, while dashed lines represent the profiles of $r_{c}=5.0 \mathrm{kpc}$ and $\alpha_{p}=2.25$. Dots are the profiles of sampled $10^{4} \mathrm{GC}$ particles that are well matched to analytical descriptions. 


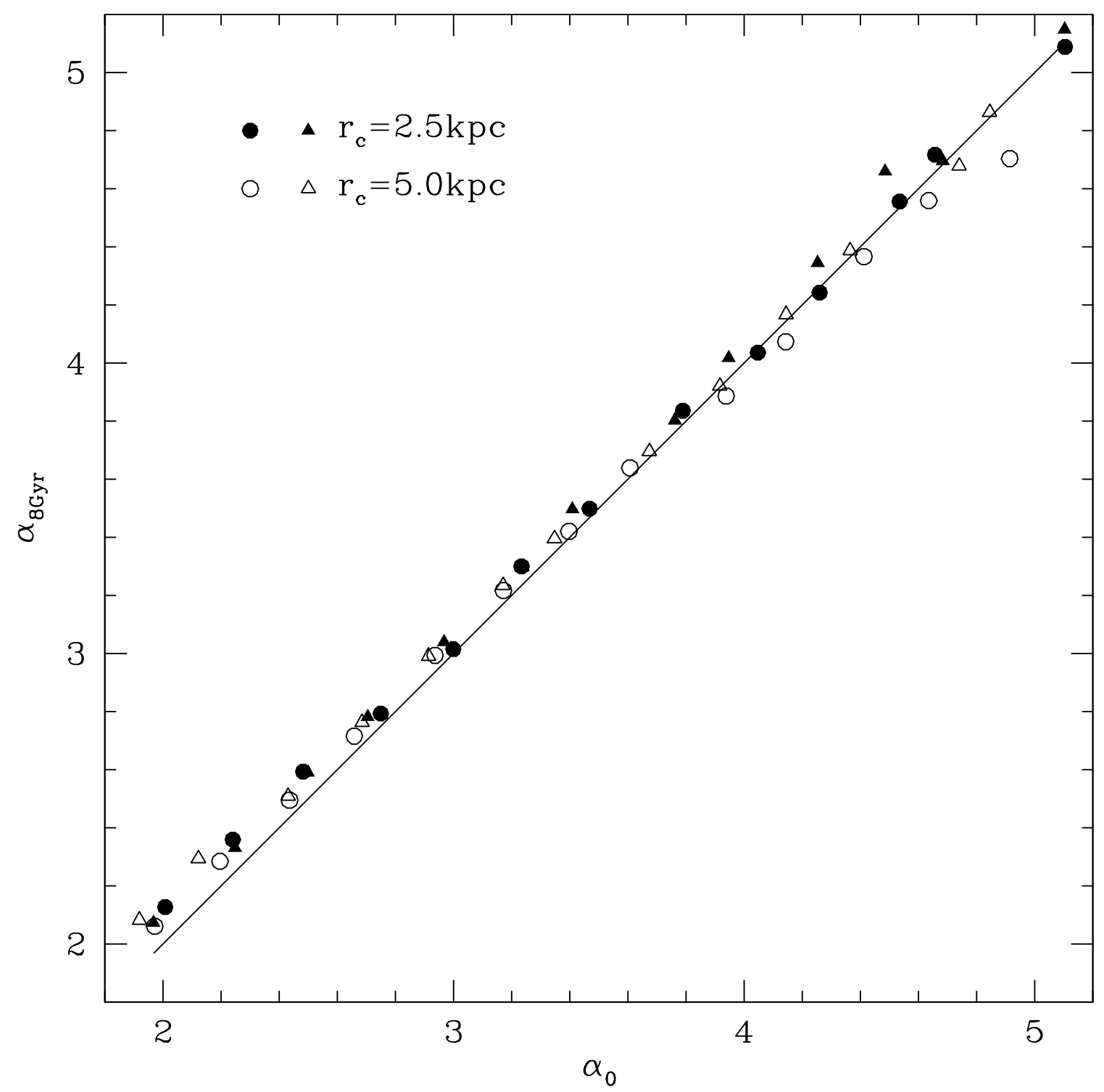

Fig. 2.- Comparison of the initial slope $\left(\alpha_{0}\right)$ with the slope $\left(\alpha_{8 G y r}\right)$ after 8 Gyrs of a simulation for an isolated galaxy without any interaction. Both high-resolution (circle) and low-resolution (triangle) cases show that the initial distributions of GCs are stable in a single isolated galaxy for 8 Gyrs. 


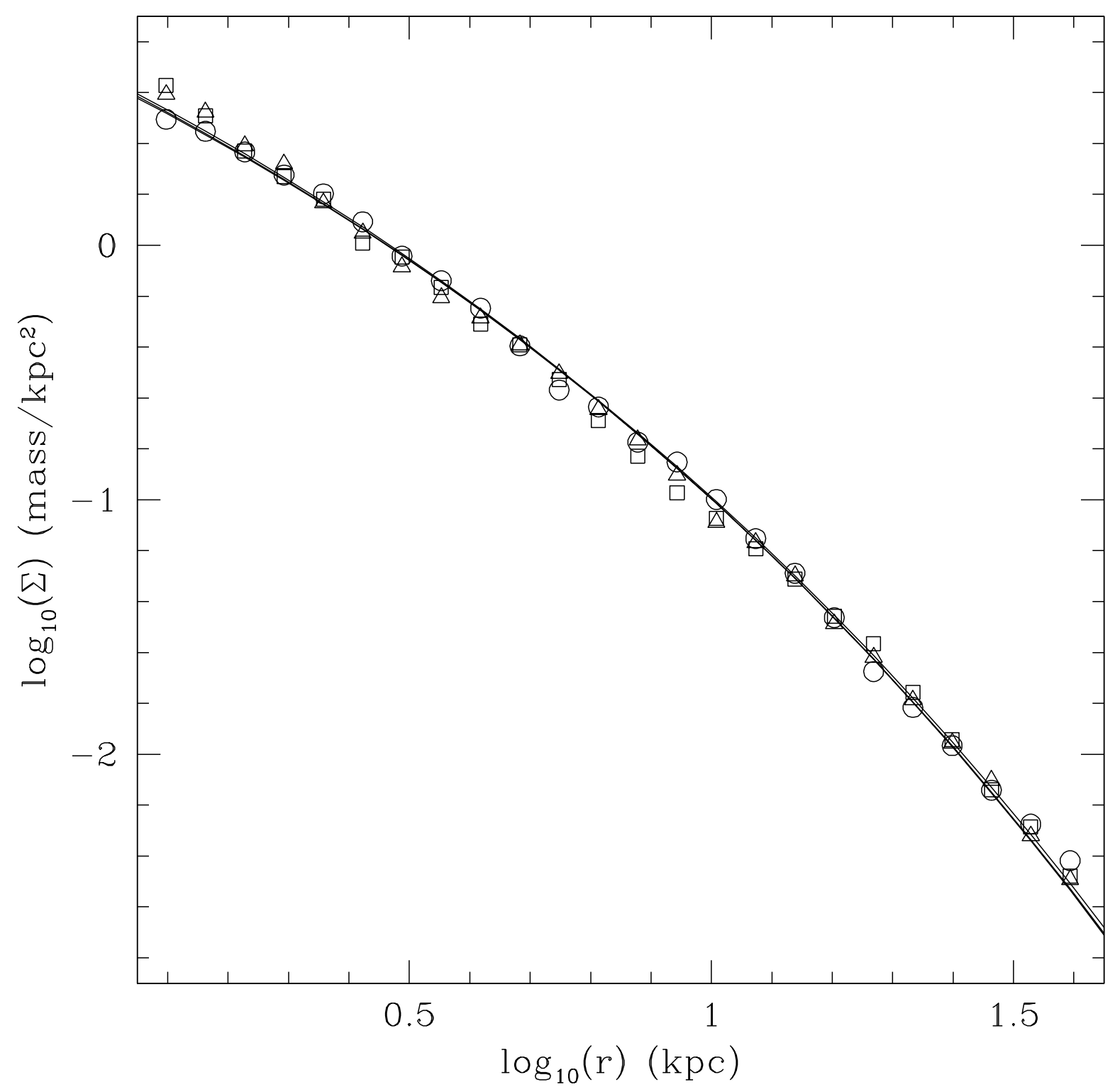

Fig. 3.- Projected mass density distribution of stars in merger remnants. The de Vaucouleurs profile (line) is well matched to stellar distributions in all merger remnants. The distributions of the simulation $\mathrm{A}, \mathrm{B}$, and $\mathrm{C}$ are represented by triangle, rectangle, and circle, respectively. The measured effective radius is about $6.2 \mathrm{kpc}$. 


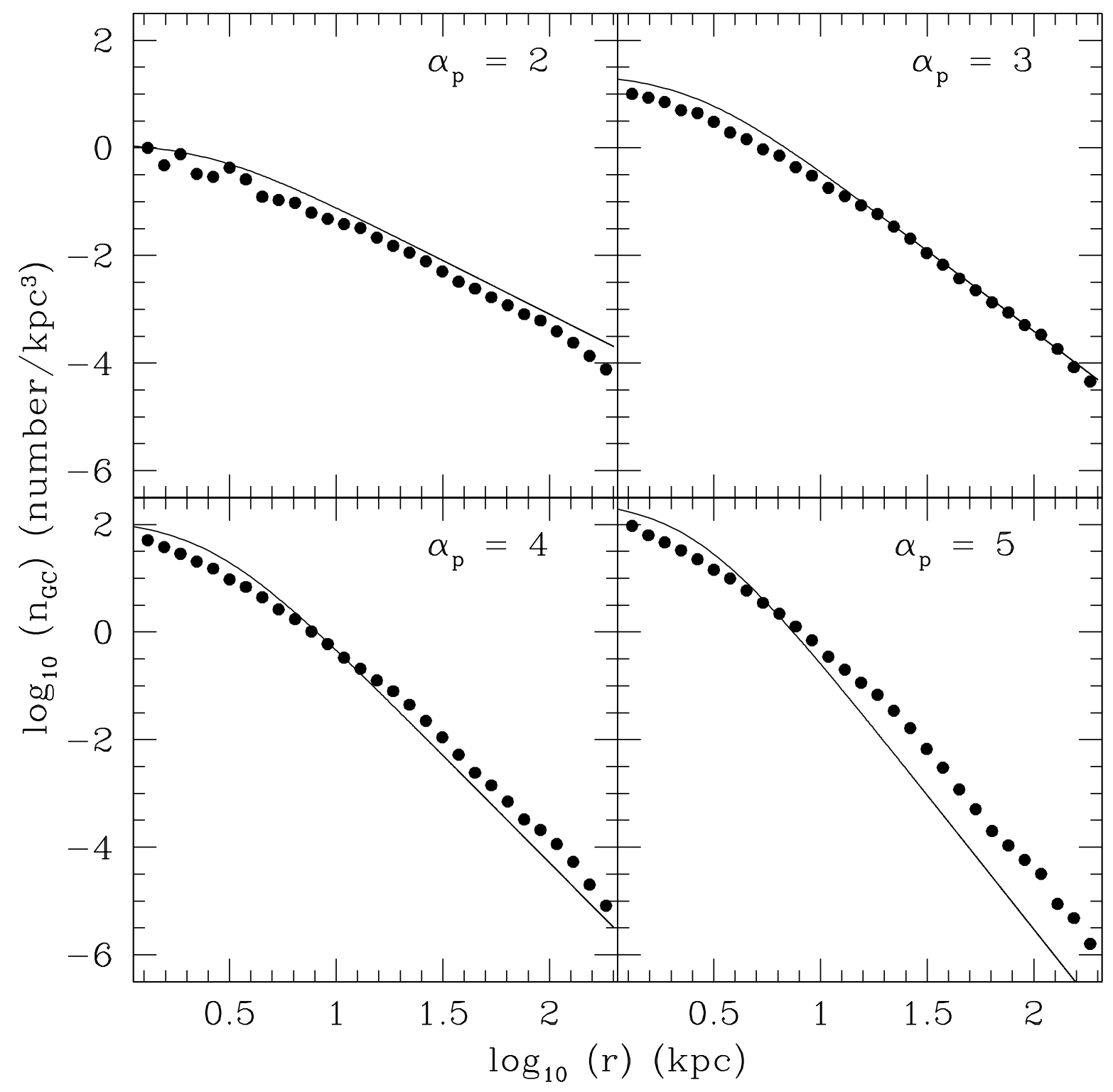

Fig. 4.- GC distributions in merger remnants for simulation B. The initial distributions of $r_{c}=2.5 \mathrm{kpc}$ are shown for $\alpha_{p}=2.0,3.0,4.0$, and 5.0 (solid line). The distributions of GCs in merger remnants are represented by dots. The initial distributions are scaled upward by a factor of two in order to match the total number of GCs in merger remnants. 

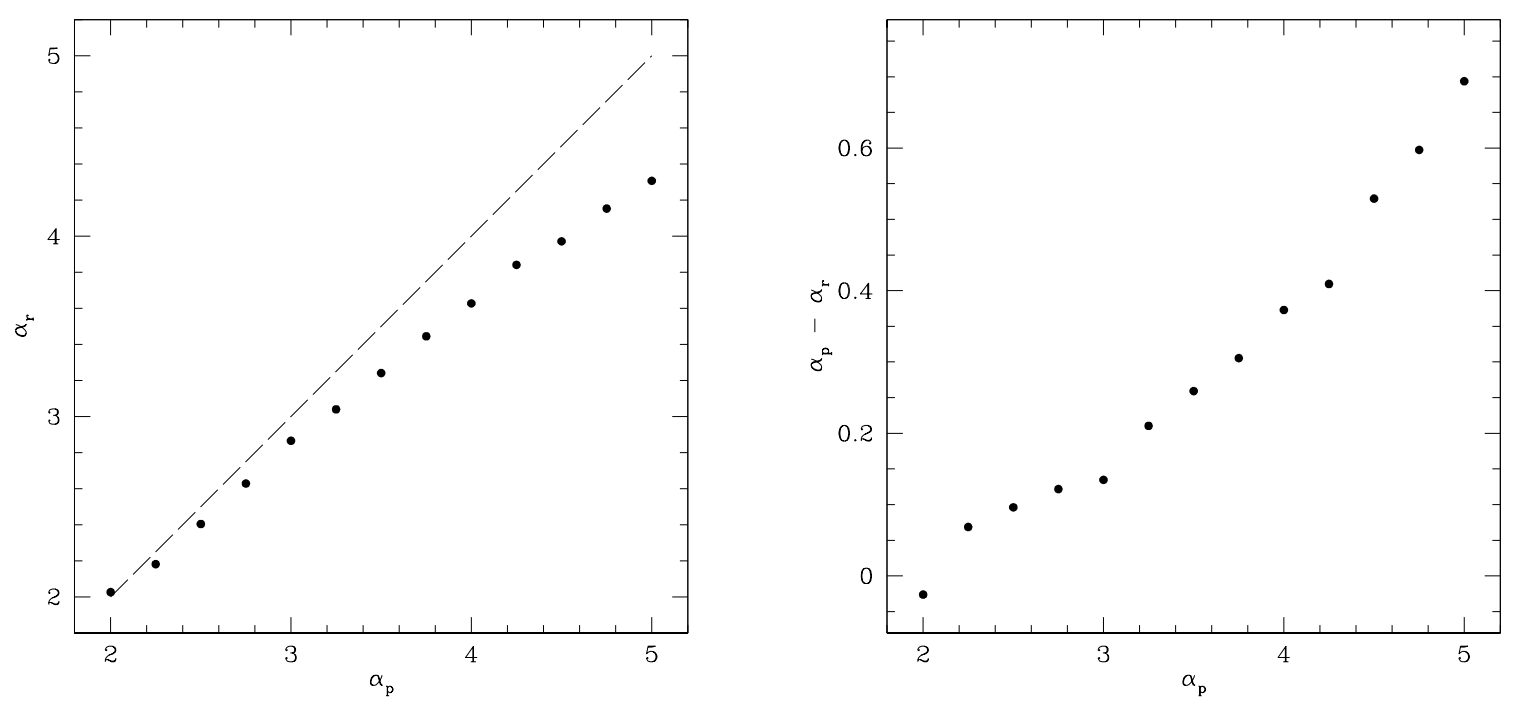

Fig. 5.- $\alpha_{r}$ (left) and $\alpha_{p}-\alpha_{r}$ (right) for simulation B with $r_{c}=2.5 \mathrm{kpc}$. The distributions in merger remnants are described as $n_{\mathrm{GC}} \propto r^{-\alpha_{r}}$ over $10<r<100 \mathrm{kpc}$. The dashed line represents no change in slope. 


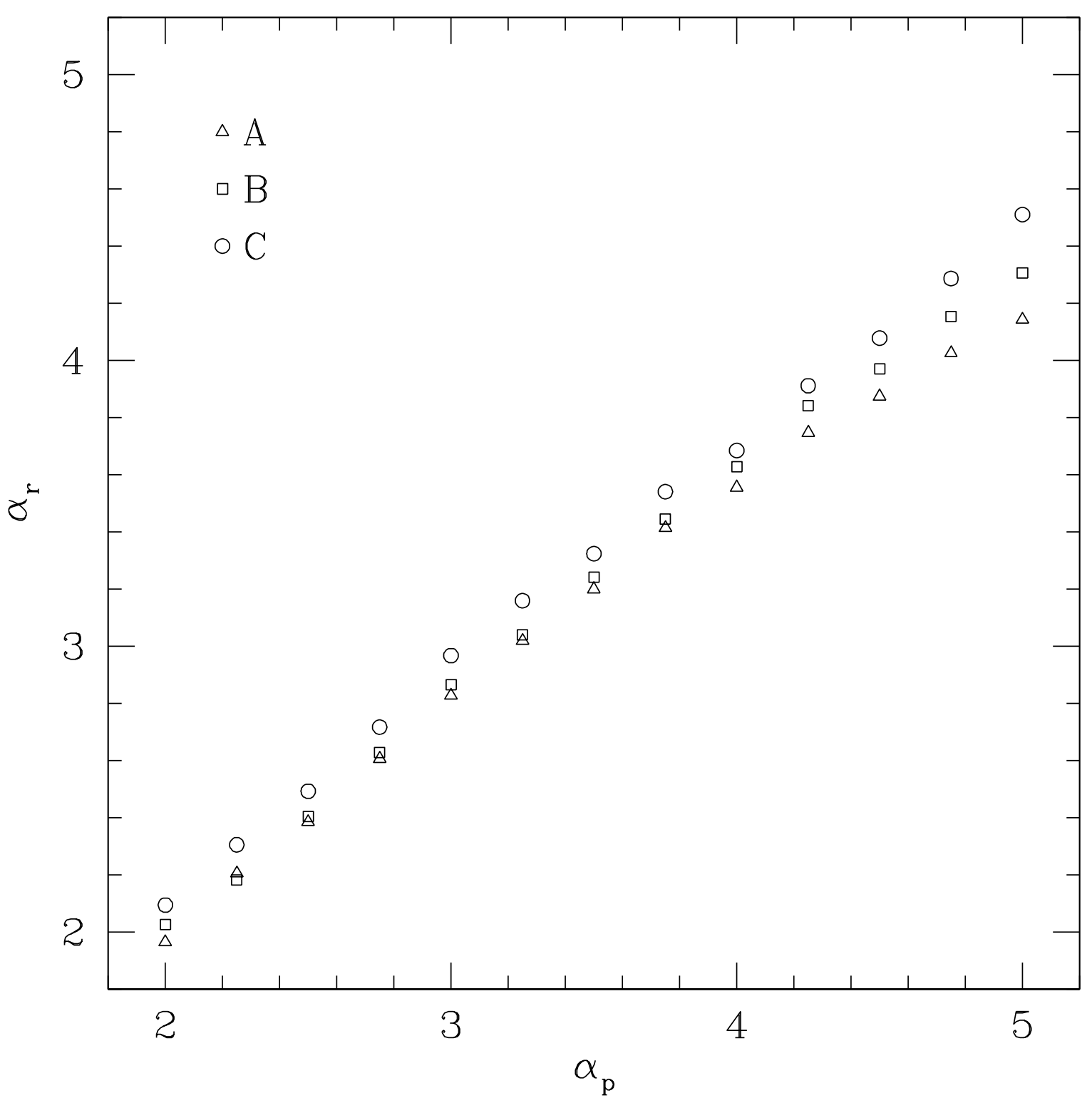

Fig. 6.- Slope of GC distributions in merger remnants produced from three different merger orbits. For the initial distribution at a fixed $r_{\mathrm{c}}=2.5 \mathrm{kpc}$, simulation C, with the highest angular momentum, shows the smallest change among the three orbits. 


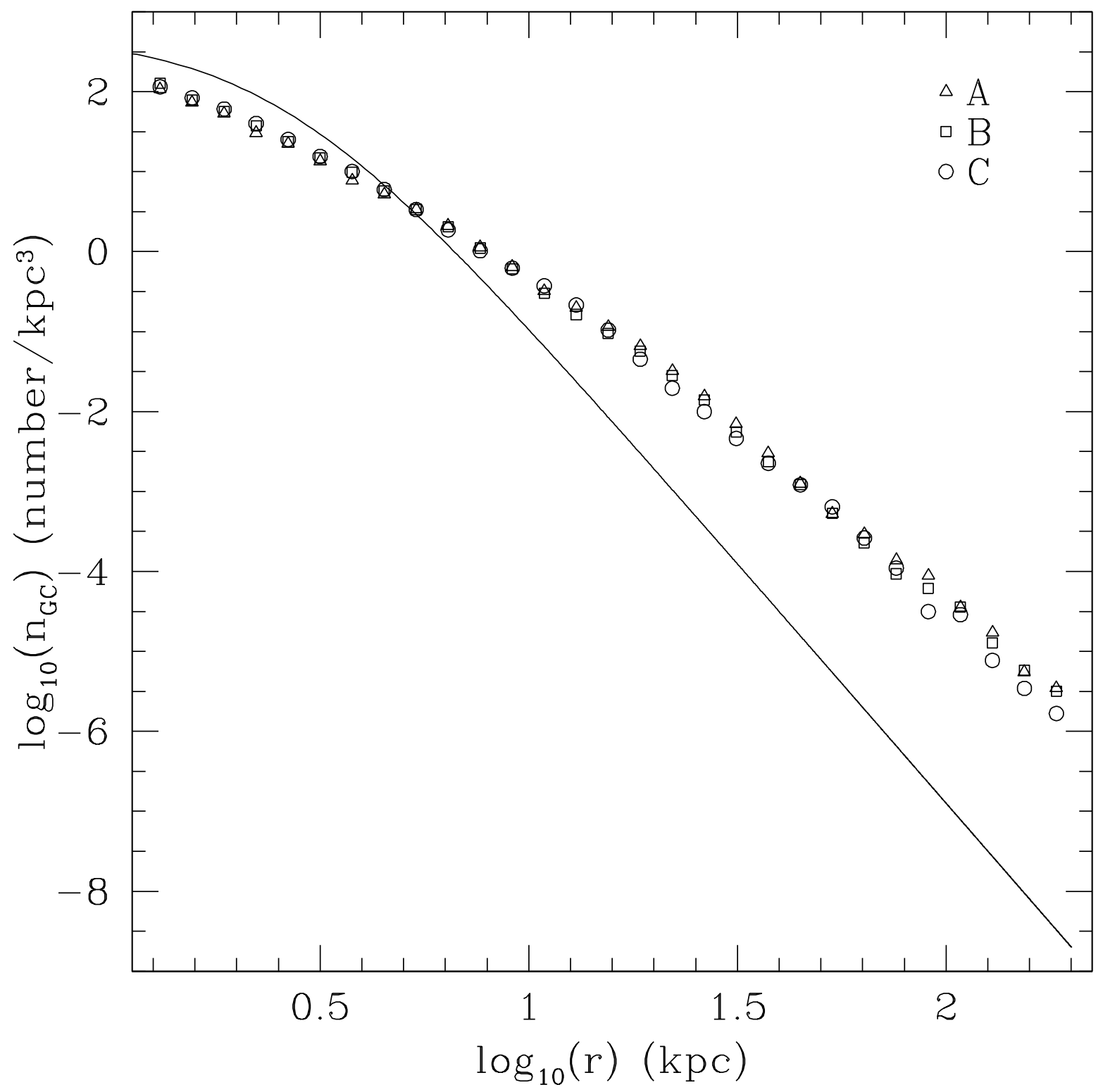

Fig. 7.- Distributions of GCs in three different merger remnants for $\alpha_{p}=5$ and $r_{c}=2.5$ kpc. The effect of different merger orbits is stronger in outer region than in inner region. The distribution in a merger progenitor is scaled upward by a factor of two for case of comparison (line). 


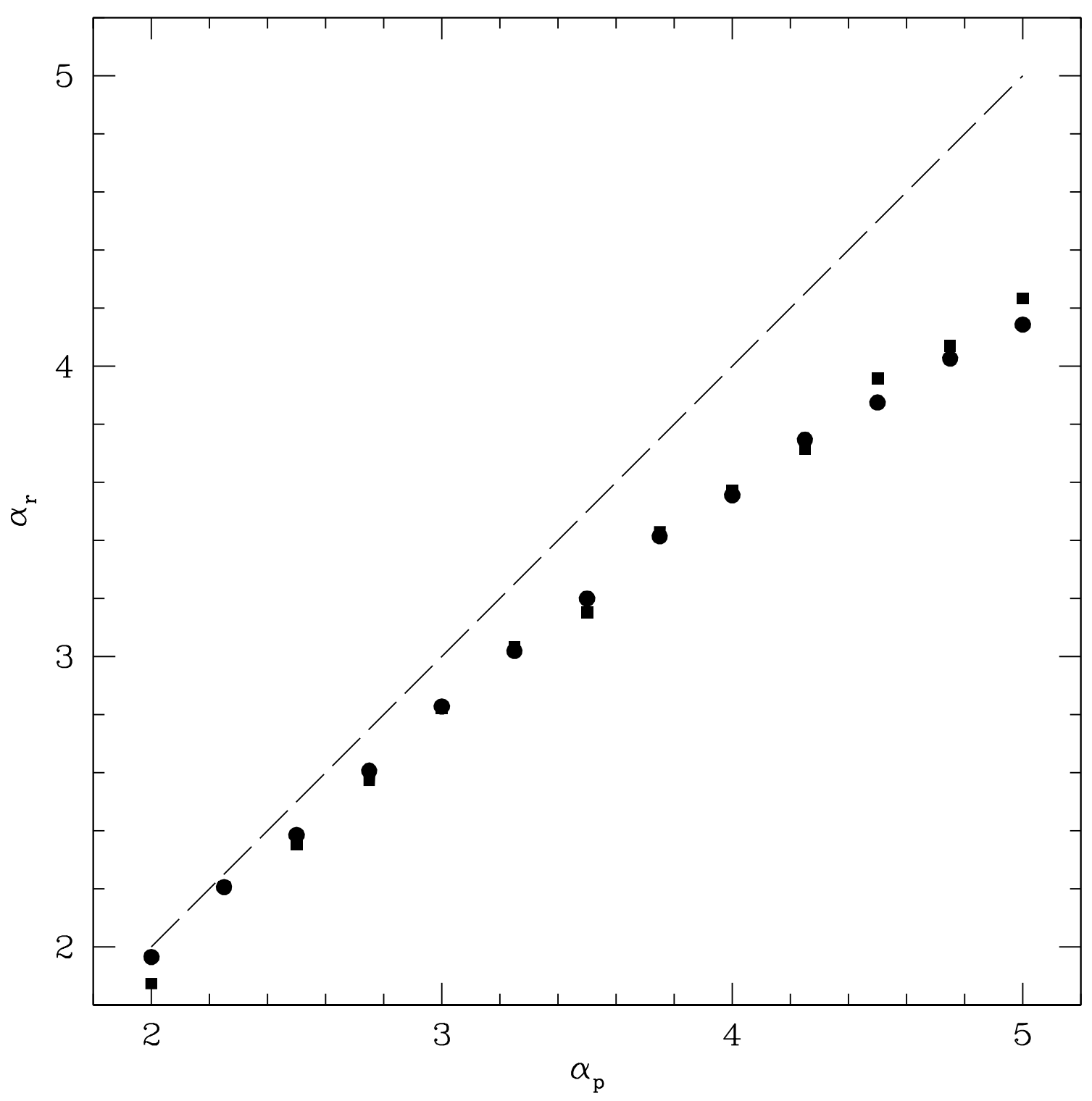

Fig. 8.- Comparison of the change of slopes $\left(\alpha_{p}\right.$ vs. $\left.\alpha_{r}\right)$ between the high-resolution (square) and low-resolution run (circle) for simulation A with $r_{c}=2.5 \mathrm{kpc}$. The change of slopes in a low-resolution run is comparable to those of a high-resolution run. 


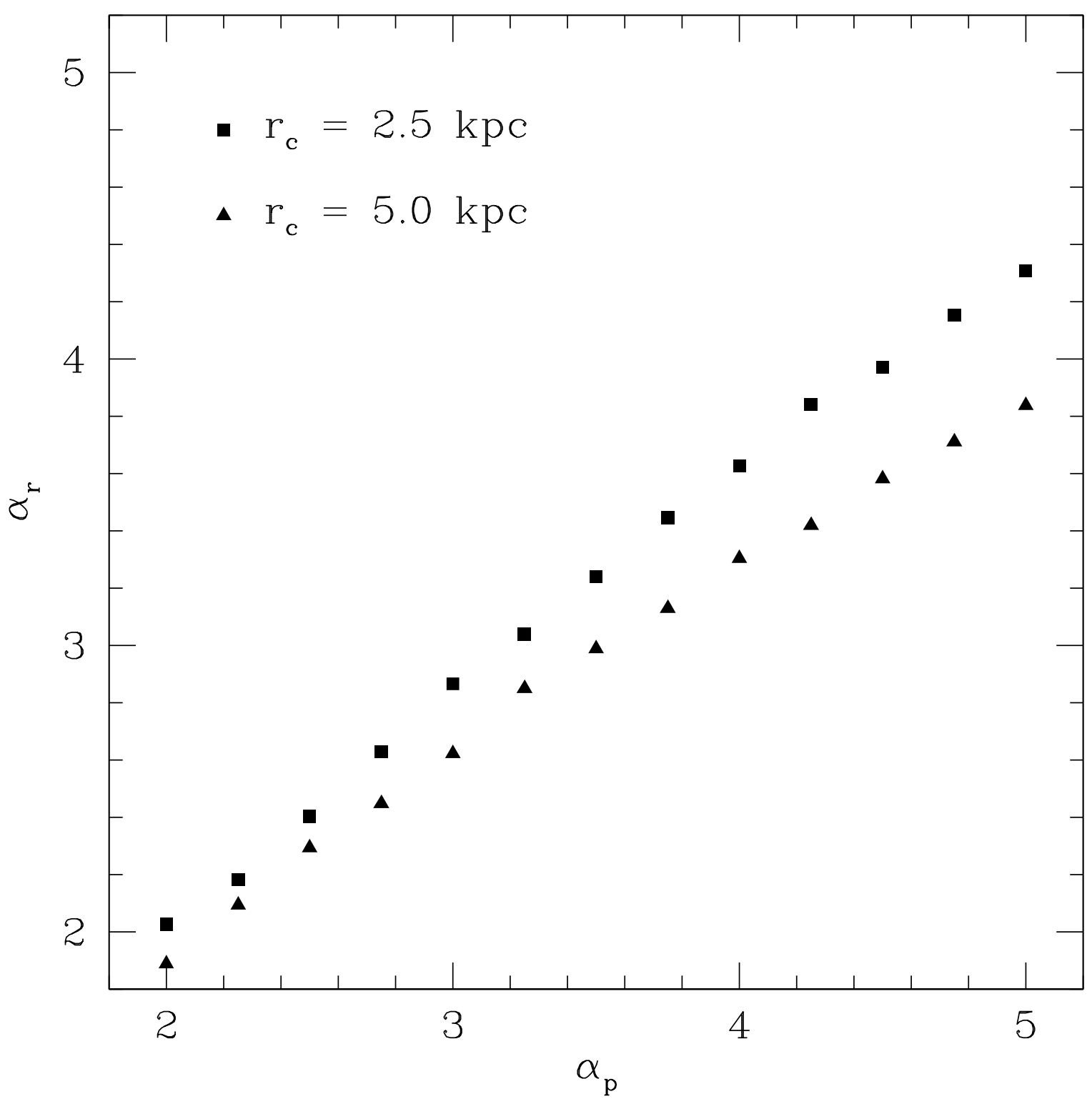

Fig. 9.- Dependence of GC distributions in merger remnants on the initial core radius, $r_{\mathrm{c}}$. Simulations B for $r_{\mathrm{c}}=2.5$ and $5.0 \mathrm{kpc}$ are compared. 


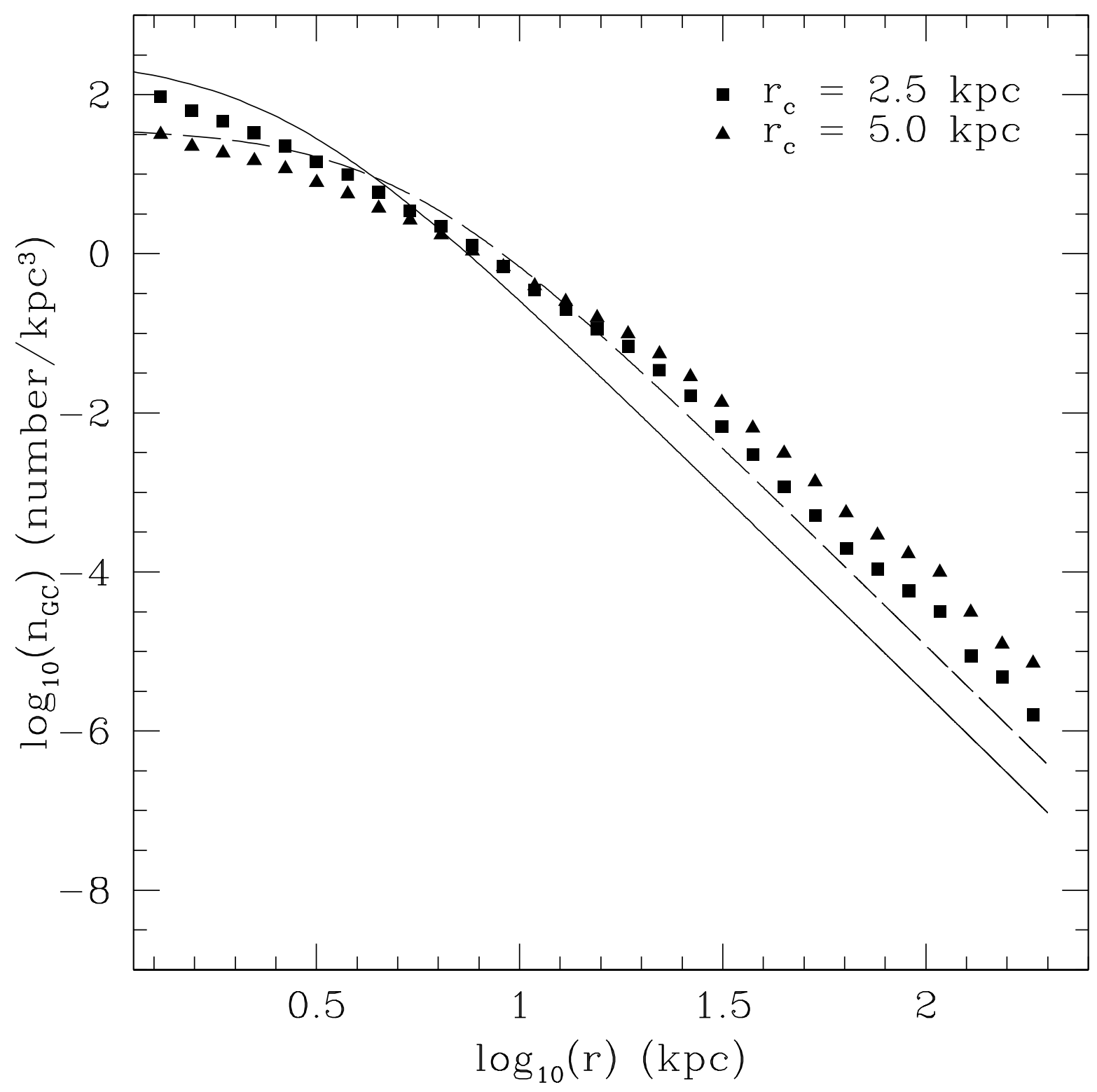

Fig. 10.- Distributions of GCs in merger remnants for $r_{\mathrm{c}}=2.5$ and $5.0 \mathrm{kpc}$. For simulation $\mathrm{B}$ and $\alpha_{\mathrm{p}}=5$, we present initial distributions in merger progenitors for $r_{\mathrm{c}}=2.5$ (solid line) and $5.0 \mathrm{kpc}$ (dashed line) and distributions in the merger remnant. 